\title{
The peculiarities of bronchopulmonary system under the influence of adverse ecological factors
}

\author{
Zhanna Abdrassulova ${ }^{1, *}$, Sultan Tuleukhanov ${ }^{1}$, Gani Issayev $^{2}$, Aidana \\ Amankeldiyeva $^{1}$, Gulmira Turmetova ${ }^{2}$ \\ ${ }^{1}$ Al-Farabi Kazakh National University, al-Farabi Ave. 71, 050040 Almaty, Republic of Kazakhstan \\ ${ }^{2}$ Khoja Akhmet Yassawi International Kazakh-Turkish University, B.Sattarkhanov Ave., 29, 161200 \\ Turkestan, Republic of Kazakhstan
}

\begin{abstract}
This research gives an insight into the problem of atmospheric pollution composition and morbidity that affects the overall well-being and health state of children of Ust-Kamenogorsk. The work shows that age specific features of the respiratory system are characterized by increase in lung volume and bronchial patency trees. The specific methodology assisted to determine the increase in the patency of large and medium bronchi occurs more dynamic in boys than in girls. The bronchial obstruction was assessed by the methods of pneumotachometry with the help of "PneuMedics" (USA) device. The environmental factors of Ust-Kamenogorsk affect the state of the cardiorespiratory system of children, so level of contamination place of residence highly affects the general health state of the children at different age categories and anthropometric measurements. One of the important results was that children with growth and weight coefficient who have higher than the average value are characterized by a decrease of functional reserves of the bronchopulmonary system, and children with the coefficient lower than average, on the contrary, are characterized by decrease in the cardiovascular system.
\end{abstract}

\section{Introduction}

More than 80 chemical compounds in the form of dust, gaseous and aerosol fractions, including the metals of the 1 st class dangers have been found in atmosphere of UstKamenogorsk, which is among the most polluted cities in the world [1]. Long-term system analysis of atmospheric pollution composition and morbidity, conducted by the office of Public health-epidemiological surveillance, revealed a clear correlation of cause and the effect of the level of pollution on the health of the population [2]. With the observed increase in pollution, the indicators of General morbidity for the period from 2000-2001 increased from 1489.0 to 1512.5. Level of cancer the incidence in the city of Ust-Kamenogorsk has increased from 13.0 to 17.4. According to expert data the territory of Ust-Kamenogorsk was divided into districts by degree of danger to public health. The most polluted and dangerous

*Corresponding author: Zhanna.Abdrassulova@kaznu.kz 
is the Ulba district, where the main industrial zone (OJSC Kazzinc, Ulba metallurgical factory and etc.). This area is defined by the expert Commission as "extremely dangerous zone for public health". The area Factory of silk fabrics is considered to have lesser degree of contamination, due to its remoteness and more favorable wind [3, 4]. Morbidity statistics also correlates with the level of pollution: in a more polluted Ulbinsk region, the incidence of the population is higher [5]. The impact of harmful factors on the maturing child's body can lead to sustainable changes in immature functional systems, subsequently, imperceptibly passing into a chronic pathology. There was revealed the dependence of functional state of the bronchopulmonary and cardiovascular system of children from the level of environmental pollution: in children living in more contaminated area, there were detected signs of reduction of functional reserves of the studied systems. The role of constitutional factor of children in the risk of diseases was determined: children who have the growth-weight ratio higher than the average value, revealed more reduction of functional reserves of the bronchopulmonary system, and children with the coefficient lower than the average had reduction of the cardiovascular system.

There have been no studies of the child population in this region. There are only a few works that have studied anthropometric data, level of physical development of children living in East Kazakhstan [6]. At the same time, the literature contains data on the study of cardiorespiratory system of children who live in the ecological disaster zone - Aral region [7], industrial region [8].

Therefore, a very important point in this case is detection of prenosological form changes before the onset of diagnosed pathological process.

According to some literature data, the functional indicators of patency the bronchial tree are very sensitive indicators of pollution atmospheric air $[9 ; 10 ; 11 ; 12]$. They reflect the reaction to changes in the external environment that characterize adaptive capabilities body, indicators of variational pulsometry according to R.M. Baevsky [13; 14].

In this regard, the purpose of our investigation was to study physiological features of the bronchopulmonary system of boys and girls of various ages living in environmentally unfavorable conditions of Ust-Kamenogorsk.

As part of this goal, the following tasks were solved:

1. Study of age and sex characteristics of the bronchopulmonary system in children living in environmentally unfavorable conditions of Ust-Kamenogorsk.

2. Comparative study of children's cardiorespiratory system, living in two different pollution areas.

3. Based on the goal and objectives of the study, we studied age and sex characteristics of bronchial patency, external hemodynamics, variational heart rate monitoring and daily monitoring ECG in children from 6 to 15 years of age living in environmentally unfavorable conditions of Ust-Kamenogorsk. The material base of the Medical Unit-2 in UstKamenogorsk, the Department of functional diagnostics, was used: with the help of peak flow meter "PneuMedics" (USA) the state of bronchial patency was determined.

\section{Materials and Methods}

The choice of methodical techniques and the volume of research were determined by the purpose and objectives of this work. In order to solve the tasks 42 children without the established pathology were examined: 21 boys and 21 girls at the ages 6-15 years, who live in Ust-Kamenogorsk. The examined children were conditionally divided into three age groups of 6-8 years, 9-12 years, 13-15 years, respectively (according to age periodization). The group №1 included children who live in less hazardous area for population, such as the 
district of the silk factory; a group №2 is represented by children who live in extremely hazardous area for population: Ulba district [4]. The studies were conducted in the department of functional diagnostics of the health unit-2 in Ust-Kamenogorsk. The temperature in the room was $18-20^{\circ} \mathrm{C}$. The examination of children was conducted in a spring-summer period.

Complex examination of children has begun with the registration of information about the state of health, overall well-being, and age. The anthropometric data were measured with the help of stadiometer and medical scales: height $(\mathrm{H}, \mathrm{cm})$; the subject was in a quiet position, straightened the chest, draw in a stomach, touching the vertical point of a stadiometer with his three points - heels, buttocks, shoulder blades. The head was in the position where the outer corner of an eye and an external auditory meatus were on one level. Body mass (m, $\mathrm{kg}$ ); the subject stood in the middle of the scale, the weighing accuracy was $\pm 50 \mathrm{~g}$.

Functional features of cardiorespiratory system were evaluated by methods of pneumotachometry, variational pulsometry, electrocardiography, Holter daily monitoring of electrocardiography. According to the methodical approach, the studies were carried out in a position of relative rest of the examined children.

The bronchial obstruction was assessed by the methods of pneumotachometry with the help of "PneuMedics" (USA) device according to the following indicators: FVC - forced lung capacity (1), FEV1 - volume of forced air in a second (1), Peak Flow - peak volume velocity (1/s), FEF 25-75 - average maximum volume velocity (1/s), FEF (MOC)-75 maximum volume velocity on the level of small bronchi (1/s), FEF (MOC)-50 - maximum volume velocity on the level of middle bronchi (1/s), FEF (MOC)-25 - maximum volume velocity on the level of large bronchi (1/s), SVC - vital lung capacity (1), ERV - respiratory volume (1), IC - reserve inspiratory volume (1), \%FEF25-75 - permeability ratio of large, middle and small bronchi relatively to Central European standards (\%).

Testing of the individual characteristics was determined with the help of following methods.

Determination of cardiorespiratory reserve, bar test. The subject of study, who was in a rest condition, sitting, was measured for the heart rate frequency with the stopwatch. Breath was held after taking a deep breath. The time of maximum breath holding was measured $(\mathrm{MBH})$. Right after breathing resume, the HRF was measured repeatedly in 10 seconds.

\section{Results and Discussion}

According to the research objectives, the results are presented the three corresponding sections of this chapter. The first section includes results of the study of age and gender characteristics of children living in Ust-Kamenogorsk. The second section includes comparative results of examination of the cardiorespiratory system children living in areas with different levels of pollution: more polluted - Ulba district and less polluted- silk factory region. The third section is devoted to identifying the features of response methods cardiorespiratory system and the degree of stability of the body to exposure to adverse factors depending on some individual characteristics of the child.

Anthropometric data. Results of anthropometric measurements presented in table 1, they correspond to age-related patterns of growth and development. It can be noted that boys and girls of the first and second age groups have the same length and weight indicators. At the age of 13-15 years, the length and body weight ( $\mathrm{p}: 0,05)$ higher for boys than girls, which means that a greater the rate of morphofunctional growth of boys in puberty. The difference between the length and weight of the body between the second and third the age range is $\Delta \mathrm{R}=25.15 \mathrm{~cm}, \Delta \mathrm{m}=20.43 \mathrm{~kg}$ for boys and $\Delta \mathrm{R}=14.75 \mathrm{~cm}, \Delta \mathrm{m}=8.73 \mathrm{~kg}$ for girls. Comparative information between the first and second age group: $\Delta R=20.85 \mathrm{~cm}, \Delta \mathrm{m}=11.57 \mathrm{~kg}$ allow us 
to conclude that girls puberty jump is observed in the age group of 9-12 years, apparently there are two jumps in boys, aged 9-12 and 13-15. This is consistent with a lot of literary data about earlier maturation of girls compared to boys. At the same time, it was revealed that the growth spurt includes two phases [15-17].

The growth/mass ratio $(\mathrm{P} / \mathrm{m})$ of the body tends to decrease with age, and boys in the age group of 13-15 years in the greater degree than in girls ( $\left.\mathrm{p}^{\prime \prime} 0.05\right)$, which indicates a more intense increase in height than increase in body weight according to age morpho-functional regularities .

Table 1. Anthropometric data of boys and girls of three age groups

\begin{tabular}{l|c|c|c|c|c|c}
\hline & \multicolumn{3}{|c|}{ Boys } & \multicolumn{3}{c}{ Girls } \\
\cline { 2 - 7 } & $\begin{array}{c}6-8 \text { y.o., } \\
\mathrm{n}=18\end{array}$ & $\begin{array}{c}9-12 \text { y.o., } \\
\mathrm{n}=18\end{array}$ & $\begin{array}{c}13-15 \text { y.o., } \\
\mathrm{n}=19\end{array}$ & $\begin{array}{c}6-8 \text { y.o., } \\
\mathrm{n}=21\end{array}$ & $\begin{array}{c}9-12 \text { y.o., } \\
\mathrm{n}=23\end{array}$ & $\begin{array}{c}13-15 \text { y.o., } \\
\mathrm{n}=20\end{array}$ \\
\hline Body length, cm & $122,71 \pm 2,79$ & $141,42 \pm 4,66$ & $166,57 \pm 4,63$ & $123 \pm 2,66$ & $143,85 \pm 3,73$ & $158,57 \pm 3,82$ \\
\hline Weight, kg & $26,14 \pm 1,84$ & $36,28 \pm 1,88$ & $56,71 \pm 1,70^{*}$ & $25,28 \pm 1,44$ & $36,85 \pm 2,25$ & $45,57 \pm 2,65^{*}$ \\
\hline P/m & $4,81 \pm 0,34$ & $3,92 \pm 0,15$ & $2,97 \pm 0,04^{*}$ & $4,97 \pm 0,31$ & $3,95 \pm 0,19$ & $3,54 \pm 0,19^{*}$ \\
\hline
\end{tabular}

Age and sex characteristics of the bronchopulmonary system. The obtained results of the bronchopulmonary system revealed age and gender differences. Table 2 shows that with age the volume indicators of the lungs are increasing bronchopulmonary patency of small, medium and large bronchi, which is quite a natural process of physiological growth. As expected, from the initial anthropometric data, the value of lung volumes and bronchial patency in boys 13-15 years is higher than in girls of the same age, which confirms the harmony of development functional systems in the process of growth. Moreover, the correlation analysis of indicators revealed significant correlations between indicators of height, weight, and bronchial patency $(\mathrm{p}<0.05)$. It can be noted that the indicators of patency of large bronchi boys of all ages are higher than girls of the corresponding age, while the indicators of patency of the middle and small bronchi in boys aged 6-8 years are lower than girls of the same age, but in the second and third age groups, these indicators in boys become higher than that of girls. As far as we know from previous data, anthropometric indicators for boys and girls differ only in the third age group. Accordingly, we can conclude that in boys there is a large dynamic increase in patency large bronchi with age, with some delay in the periphery bronchial tree.

Table 2. Indicators of bronchopulmonary system of boys and girls of three age groups

\begin{tabular}{l|c|c|c|c|c|c}
\hline & \multicolumn{4}{|c|}{ Boys } & \multicolumn{3}{c}{ Girls } \\
\cline { 2 - 7 } & $\begin{array}{c}6-8 \text { y.o., } \\
\mathrm{n}=18\end{array}$ & $\begin{array}{c}9-12 \text { y.o., } \\
\mathrm{n}=18\end{array}$ & $\begin{array}{c}13-15 \text { y.o., } \\
\mathrm{n}=19\end{array}$ & $\begin{array}{c}6-8 \text { y.o., } \\
\mathrm{n}=21\end{array}$ & $\begin{array}{c}9-12 \text { y.o., } \\
\mathrm{n}=23\end{array}$ & $\begin{array}{c}13-15 \text { y.o., } \\
\mathrm{n}=20\end{array}$ \\
\hline FVC & $1,19 \pm 0,17^{*}$ & $2,06 \pm 0,19$ & $3,36 \pm 0,28$ & $1,26 \pm 0,06^{*}$ & $1,73 \pm 0,14$ & $2,74 \pm 0,26$ \\
\hline FEVI & $1,17 \pm 0,16^{*}$ & $2,00 \pm 0,20$ & $3,26 \pm 0,25^{*}$ & $1,22 \pm 0,06^{*}$ & $1,68 \pm 0,13$ & $2,37 \pm 0,17^{*}$ \\
\hline PEAK FLOW & $2,38 \pm 0,33$ & $3,53 \pm 0,49^{*}$ & $5,60 \pm 0,86$ & $2,41 \pm 0,19$ & $2,93 \pm 0,11^{*}$ & $3,87 \pm 0,46$ \\
\hline FEF 25-75 & $1,77 \pm 0,22$ & $2,71 \pm 0,34$ & $4,28 \pm 0,29^{*}$ & $1,92 \pm 0,22$ & $2,37 \pm 0,15$ & $3,21 \pm 0,43^{*}$ \\
\hline FEF 25 & $2,31 \pm 0,33$ & $3,41 \pm 0,52^{*}$ & $5,34 \pm 0,79$ & $2,22 \pm 0,29$ & $2,76 \pm 0,07^{*}$ & $3,47 \pm 0,50$ \\
\hline FEF 50 & $1,94 \pm 0,22$ & $2,87 \pm 0,38$ & $4,49 \pm 0,36^{*}$ & $2,15 \pm 0,22$ & $2,62 \pm 0,17$ & $3,42 \pm 0,46^{*}$ \\
\hline FEF75 & $1,25 \pm 0,18$ & $1,95 \pm 0,20$ & $3,22 \pm 0,15^{*}$ & $1,50 \pm 0,10$ & $1,65 \pm 0,16$ & $2,45 \pm 0,39^{*}$ \\
\hline SVC & $1,49 \pm 0,18$ & $2,23 \pm 0,20$ & $3,76 \pm 0,29^{*}$ & $1,37 \pm 0,08$ & $1,96 \pm 0,15$ & $2,82 \pm 0,22^{*}$ \\
\hline ERV & $0,23 \pm 0,11$ & $0,37 \pm 0,12$ & $0,62 \pm 0,11$ & $0,20 \pm 0,08$ & $0,24 \pm 0,10$ & $0,68 \pm 0,15$ \\
\hline IC & $1,26 \pm 0,18$ & $1,85 \pm 0,11$ & $3,14 \pm 0,23^{*}$ & $1,17 \pm 0,11$ & $1,72 \pm 0,11$ & $2,14 \pm 0,12^{*}$ \\
\hline
\end{tabular}

Note: significant sex differences within the same age $\left(\mathrm{p}<0,05^{*}\right)$ 
Comparative percentage of traffic indicators bronchial tree with Central European standards revealed following regularities. The patency of the major bronchi in the examined children of all age groups are below the standards, which is more degree expressed in girls $(91.14 \pm 9.73 ; 86.42 \pm 11,07 ; 85,71 \pm 10,57$ - for boys; $74 \pm 10,35 ; 74,71 \pm 4,71 ; 70 \pm 6,02$ - for girls). The percentage of patency of the middle bronchi is also lower than $100 \%$ in the first and second age groups and higher in the third age group $(98,42 \pm 5,98 ; 95,14 \pm 12,29$; $104,28 \pm 9,15$ - in boys, $92,80 \pm 13,14 ; 89 \pm 2,70 ; 102.33 \pm 7.22$ - in girls). As for the small bronchi, it is observed the opposite picture: the percentage ratio is higher-100\% in almost all children, except for the group of girls aged 9-12 years $(107.71 \pm 9.38 ; 115.14 \pm 16.18$; $130.42 \pm 8.06$-for boys: $120,8 \pm 10.24 ; 98 \pm 5,31 ; 137,33 \pm 13,53$ - girls), Revealed differences in the rate of patency of the bronchial tree can be a consequence of both climatic and geographical features areas where the children being examined live, as well as unfavorable ones ecological impact. To clarify this issue, we conducted comparative analysis of data from two groups of children living in the same climatic and geographical conditions, but with different levels of pollution. This data is presented in the next chapter.

Thus, the patency of the bronchial tree has age-related changes in accordance with the laws of growth and development: with age there is an increase in the patency of large, medium and small bronchi in all children. Increased patency of large and medium-sized bronchi occurs more dynamically in boys than in girls, according to anthropometric data.

Analysis patency of the bronchi the tree is relative Central European standards have shown that the patency of large bronchi is lower than the standards, medium bronchi is close to them, and small bronchi - a little higher: this may be due to the impact of environmentally adverse factors on the bronchopulmonary system of the examined children.

It is necessary to pay attention to the fact that this is expressed in girls more than boys, indicating greater sensitivity girls'. This is also confirmed by the above data on volume: lung function.

Age and sex characteristics of the cardiovascular system. Blood pressure and heart rate indicators abbreviations at rest

The reaction of the HRF and the maximum delay of breathing on bending the torso (Belgian test). Indicators of the health coefficient (according to R. M. Baevsky) as a characteristic of the adaptive potential of the circulatory system.

Heart rate and blood pressure values in three age groups of boys and girls correspond to the norm; there are no significant differences between pears detected. Only in the group of 9-12 years the ADF index in boys significantly higher than in girls.

Sexual differences were found in the response of the heart rate to the maximum breathholding. In boys of the first and third age groups the PR1 index is significantly higher than that of girls. You should pay attention to the duration of maximum breath retention. You can see that in the group of boys 6-8 years of age, the duration of $\mathrm{MBH}$ is the lowest $(18,14 \pm 2,94$ $\mathrm{s})$, and in the group boys 13-15 years of age the highest (65.71 $\pm 7.27 \mathrm{~s})$, a higher value of PR1 (but still normal to 1,2) and the older age group can be explained a sufficiently long delay time (almost twice as long as the girls of the appropriate age and three times as much as the younger group boys').

Whereas the combination of the minimum value of $\mathrm{MBH}$ and a high index of PR1 (above 1.2) in boys of the younger group is likely indicates a decrease in the cardiorespiratory reserve.

At the same time, indicators of the cardiovascular system's response to the torso tilts showed no significant differences. Displays PR2 in all groups correspond to the norm and have similar values almost in children's. The lowest values of PR2 in girls 9-12 years old differ significantly from the corresponding values in boys. Values of the health coefficient 
that characterize the adaptive potential of the circulatory system have a level of "satisfactory» adaptation (close to 2) in all children. You can note, that this indicator is relatively better in the group of girls 9-12 years old (lowest p value: 0.05 ).

Thus, according to the above data, we can say that the most optimal indicators that characterize the heart rate-vascular system, observed in girls 9-12 years. It is possible that girls in puberty there is a large optimization of the studied indicators. However, the PR1 index of this group of girls indicates a decrease in the cardiorespiratory reserve (more than 1.2). Given that the duration of maximum breath retention is the second the age group is longer (45 min, which is defined as "satisfactory" cardiorespiratory reserve). Than in others age groups, we believe that after all, for a more correct interpretation of the indicator PR1 should be performed breath holding with the specified time, or to calculate the PR based on delay time. For example PR1 in girls of the first and third groups indicates optimal cardiorespiratory reserve (below 1.2) at the same time; the duration of maximum breath retention indicates an "unsatisfactory" level of cardiorespiratory reserves.

Comparative studies of indicators of the cardiorespiratory system in children living in two districts with different degrees of pollution. Anthropometric data for boys and girls living in two different regions of pollution are presented in table 3 . It can be seen that boys of group 2 have higher growth than boys of the control group $(\mathrm{p}<0.05)$. Girls have no significant differences in anthropometric data. It should be noted that the $\mathrm{P} / \mathrm{m}$ coefficient has significant differences between the two groups in boys, while girls do not have such differences.

Table 3. Anthropometric data for boys and girls living in the area (1) and (2)

\begin{tabular}{l|c|c|c|c}
\hline & Boys 1 & Boys 2 & Girls 1 & Girls 2 \\
\hline Height $(\mathrm{cm})$ & $137,77 \pm 6.17^{*}$ & $147,9 \pm 6,54^{*}$ & $142,22 \pm 5,81$ & $141,5 \pm 5,17$ \\
\hline Weight $(\mathrm{kg})$ & $39,55 \pm 4,16$ & $39,83 \pm 4,44$ & $35,11 \pm 2,81$ & $36,55 \pm 3,33$ \\
\hline P/m & $3,67 \pm 0,23^{*}$ & $4,07 \pm 0,31$ & $4,17 \pm 0,24$ & $4,14 \pm 0,28$ \\
\hline \multicolumn{2}{ll}{ Note: significant differences within the same sex $(\mathrm{p}<0.05 *)$} \\
\hline
\end{tabular}

Note: significant differences within the same $\operatorname{sex}(\mathrm{p}<0.05 *)$

Indicators of bronchopulmonary system in children in two areas. The results of the examination of the bronchopulmonary system revealed differences that correspond to the constitutional features of two heterogeneous groups of boys. This is explained by their higher growth, which is confirmed by the revealed positive correlation between the growth characteristics and lung volume indices (0.74-0.93). In this case, we cannot say about the influence of environmentally unfavorable factors, since the groups initially had heterogeneous anthropometric data. It can be concluded that the development of the bronchopulmonary system is harmonious, in accordance with the growth and body weight in both groups of boys examined (table 4).

Table 4. Indicators of the bronchopulmonary system of boys and girls living in the area (1) and (2)

\begin{tabular}{l|c|c|c|c}
\hline & Boys 1 & Boys 2 & Girls 1 & Girls 2 \\
\hline FVC & $1,92 \pm 0,29^{*}$ & $2,41 \pm 0,34^{*}$ & $1,92 \pm 0,26$ & $1,90 \pm 0,23$ \\
\hline FEV1 & $1,88 \pm 0,28^{*}$ & $2,34 \pm 0,32^{*}$ & $1,75 \pm 0,19$ & $1,76 \pm 0,18$ \\
\hline Peak Flow & $3,15 \pm 0,47^{*}$ & $4,36 \pm 0,67^{*}$ & $2,81 \pm 0,19^{*}$ & $3,26 \pm 0,31^{*}$ \\
\hline SVC & $2,14 \pm 0,33^{*}$ & $2,76 \pm 0,34^{*}$ & $2,02 \pm 0,22$ & $2,07 \pm 0,23$ \\
\hline ERV & $0,32 \pm 0,12^{*}$ & $0,47 \pm 0,02^{*}$ & $0,35 \pm 0,14$ & $0,39 \pm 0,10$ \\
\hline IC & $1,82 \pm 0,26^{*}$ & $2,28 \pm 0,29^{*}$ & $1,69 \pm 0,13$ & $1,67 \pm 0,17$ \\
\hline FEF25-75 & $2,47 \pm 0,40^{*}$ & $3,26 \pm 0,37^{*}$ & $2,34 \pm 0,17$ & $2,62 \pm 0,33$ \\
\hline FEF25 & $3,05 \pm 0,47^{*}$ & $4,16 \pm 0,63^{*}$ & $2,72 \pm 0,15$ & $2,89 \pm 0,36$ \\
\hline FEF50 & $2,65 \pm 0,41^{*}$ & $3,44 \pm 0,40^{*}$ & $2,54 \pm 0,19$ & $2,88 \pm 0,33$ \\
\hline
\end{tabular}




\begin{tabular}{l|c|c|c|c}
\hline FEF75 & $1,86 \pm 0,32^{*}$ & $2,35 \pm 0,26^{*}$ & $1,71 \pm 0,14$ & $1,99 \pm 0,27$ \\
\hline \multicolumn{4}{l}{ Note: significant differences within the same sex $\left(\mathrm{p}<0.05^{*}\right)$} \\
\hline
\end{tabular}

At the same time, in girls where there are no significant differences in anthropometric indicators, the opposite trend is observed: in the group of girls living in a more polluted area, lung volume indicators are less than in the group of subjects living in a less polluted area, which certainly indicates a harmful influence of the external environment. And probably, we can talk about a greater reactivity of the female body to harmful environmental conditions.

A similar pattern is observed in the analysis of bronchial patency. Indicators of bronchial patency in group 2 boys are significantly higher than in group 1 boys $(p<0.05)$. Girls have the same tendency, but less pronounced. This is an expected result according to anthropometry. It should be noted that the patency of small bronchi is higher than the average European standards for both boys and girls (100-120\%), while the patency of large bronchi is significantly lower $(\mathrm{p}<0.05,70-80 \%)$ than the average European standards for both boys and girls. These data indicate that the sign of bronchodilation of medium and small bronchi as a compensatory response to the narrowing of large bronchi relative to Central European standards may be a specific mechanism for adaptation to environmental impacts. The sign of bronchodilation is more pronounced in children living in a more polluted region.

\section{Conclusions}

As a result of complex ecological and physiological studies age, gender, regional and constitutional factors were identified features of the cardiorespiratory system of children living in Ust-Kamenogorsk:

1. Age specific features of the respiratory system are characterized by increase in lung volume and bronchial patency trees. The increase in the patency of large and medium bronchi occurs more dynamic in boys than in girls.

2. High values of the stress index in all children indicate that excessive the tension of regulatory systems correspond to "poor adaptation".

3. It is established that environmental factors of Ust-Kamenogorsk affect the state of the cardiorespiratory system of children. The nature and extent of this impact depends on the level of contamination place of residence.

4. It is shown that the children's body's resistance to the external environment depends on the constitutional features: children with growth and weight coefficient is higher than the average value are characterized by a decrease functional reserves of the bronchopulmonary system, and children with the coefficient lower than average, on the contrary, are characterized by decrease in the cardiovascular system.

\section{References}

1. Ministry of Environmental Protection in Parliament, Ecology and Sustainable Development (2003)

2. V.N. Goldobin, R.B. Shin, R.A. Muzafarov, T.I. Muzafarova, VKGU, 180-187 (2002)

3. L.G. Savelyeva, Physiological justification of the problem of adaptation of a growing organism in various regions of East Kazakhstan, 19 (1997)

4. V.L. Kozhar, Map of the state of habitats and health of the population of the East Kazakhstan region, (1991)

5. G.A. Sadykova, A.M. Kustubaeva, Zh.A. Baydeldinova etc., Bulletin of the KazNU. Series of environmental, 2 (9), 74-79 (2001) 
6. L.G. Savelyeva, N.A. Agadjanyan, VKGK, 90-103 (2002)

7. N.A. Agadjanyan, A.E. Severin, The reserves of the cardiorespiratory system and environmental conditions, 112 (BGAFK, Volgograd, 1999)

8. E.M. Kazin, H.G. Blinova, T.V. Dushenina, A.R. Galeyev, Human physiology, 1, 7076 (2003)

9. E.V. Sokolov, Human physiology, 27, 13-22 (2001)

10. N.A. Agadzhanyan, P.I. Terekhin, Human Physiology, 28(3), 112-122 (2002)

11. L.Kh. Bragin, A.G. Goncharova, Human physiology, 27, 102-105 (2001)

12. S.G. Krivoshchekov, Human Physiology, 17(1), 102-105 (1998)

13. R.M. Bayevsky, O.I. Kirillov, P.3. Kletsky, Mathematical analysis of changes in heart rate during stress, 207 (Nauka, Moscow, 1984)

14. M.P. Sapin, Z.G. Bryskina, Anatomy and physiology of children and adolescents, 455 (Academy, Moscow, 2000)

15. Zh.B. Sabyrbek, O.M. Alekseeva, S.T. Tuleukhanov, Yu.A. Kim, Bulletin of Experimental Biology and Medicine, 152(3), 353-356 (2012)

16. A.A. Mautenbaev, G.K. Atanbaeva, E.A. Kyrbassova et al., Journal of Pharmaceutical Sciences and Research, 10(12), 3214-3215 (2018)

17. J. Tanner, Growth and Constitution of human, 366 (Human biology, Moscow, 1979) 